\title{
Teenager trotz Abschreckung schwanger
}

\author{
Um Teenager-Schwangerschaften zu verhindern, setzen manche Pädagogen auf Programme, in denen \\ Mädchen eine Elternschaft simulieren. Wie wenig dies nützt, zeigt eine Evaluation aus Australien.
}

_ In dem Programm „Virtual Infant Parenting“ (VIP) wurden zwischen 2003 und 2006 in Western Australia 13- bis 15-jährige Schülerinnen in Kleingruppen zum Thema Teenager-Schwangerschaft geschult. Von Freitagnachmittag bis Montagmorgen mussten sie sich zudem um eine Puppe kümmern, die die wesentlichen Eigenschaften eines Babys simulierte. So sollten sie die Belastung und die Verantwortung kennenlernen, die eine Mutterschaft mit sich bringt.

57 der eingeladenen 66 weiterführenden Schulen der Metropolregion Perth nahmen an der Studie teil. Randomisiert genossen 1.267 Mädchen das VIP-Programm, 1.567 die übliche Gesundheitsaufklärung in der Schule. Primäres Studienziel waren die über Datenbanken erfassten Lebend- und Totgeburten sowie die induzierten Aborte bei den Teilnehmerinnen bis zum Jahr 2010.

Der Schuss ging nach hinten los: In der Interventionsgruppe kam es bei 97 Mädchen (8\%) zu mindestens einer Geburt, in der Kontrollgruppe nur bei 67 Mädchen (4\%). Auch bei Abtreibungen als erstem Schwangerschaftsereignis fand sich ein signifikanter Unterschied von 113 (9\%) vs. 101 (6\%). Nach Korrektur für Störfaktoren lag das Risiko für eine Teenager-Schwangerschaft in der VIP-Gruppe um 36\% höher.

- Brinkmann SA, Johnson SE, Codde JP et al. Efficacy of infant simulator programmes to prevent teenage pregnancy: a school-based cluster randomised controlled trial in Western Australia. Lancet. 2016;388:2264-71

\section{KOMMENTAR}

Das Ergebnis ist mit Vorsicht zu genieBen, da zwar die meisten Schulen, aber auf der individuellen Ebene nur die Hälfte der eingeladenen Schülerinnen teilnahmen. Auch konnten Spontanaborte, die gerade in dieser Altersgruppe nicht selten sind, nicht erfasst werden. Zudem war der sozioökonomische Status in der Kontrollgruppe höher.

Trotz alledem ist es doch ziemlich deprimierend, dass eine derart aufwändige Intervention sogar zu einer Zunahme unerwünschter Ereignisse führt. Damit dürften die zahlreichen Versuche, mithilfe simulierter Elternschaft TeenagerSchwangerschaften zu verhindern, wohl gestorben sein. Es ist ja auch nicht ausgeschlossen, dass manche Mädchen von der Betreuung eines Puppen-Säuglings so angetan sind, dass sie sich das RealLife-Ereignis geradezu herbeisehnen.

Prof. Dr. med. H. S. FüeßI

\section{Schwindel und Augenzittern im Kernspintomografen}

Ein gesunder 33-jähriger Mann nahm an einer klinischen Studie teil, die eine Untersuchung in einem 7-Tesla-Kernspintomografen beinhaltete. Kurz nachdem er im Scanner lag, verspürte der Mann

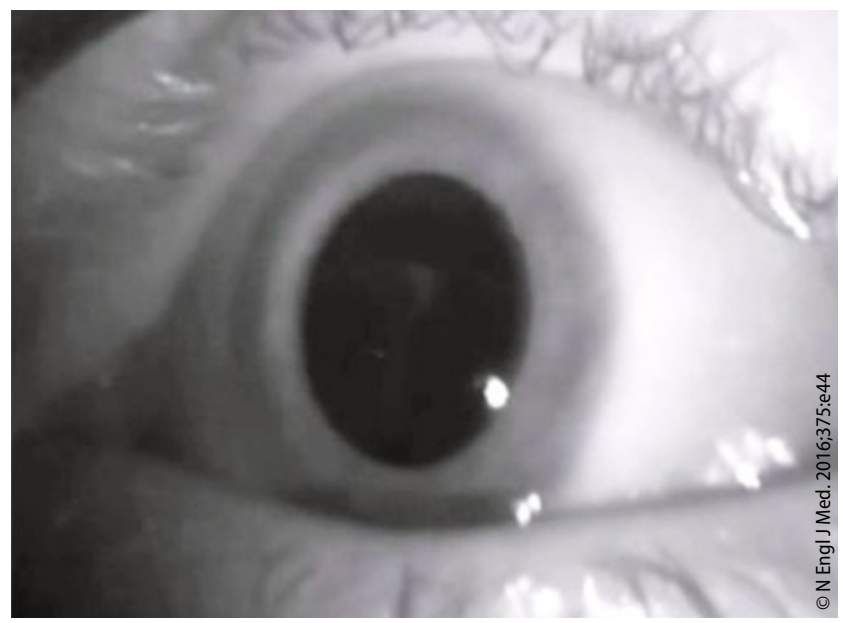

Nystagmus während der MRT-Untersuchung. für die Dauer von etwa zwei Minuten einen kurzen Drehschwindel, der unabhängig davon persistierte, ob die Augen offen oder geschlossen waren. Mit der Infrarot-Videobrille stellte man einen Nystagmus fest, der über die gesamte 90-minütige Untersuchungsdauer hinweg anhielt.

Eine Labyrinthreizung im Zusammenhang mit einer Magnetresonanztomografie ist ein bekanntes Phänomen, das durch eine Interaktion zwischen der Flüssigkeit des Innenohres und den Kräften eines statisch-magnetischen Feldes hervorgerufen wird. Die dabei auftretenden Gefühle werden von Personen unterschiedlich beschrieben und reichen von leichter Benommenheit bis zu regelrechtem Schwindel. Auch das Betriebspersonal dieser Anlagen hat gelegentlich derartige Beschwerden.

Nach dem Verlassen des Kernspintomografen fühlte sich der Proband noch einige Minuten schwindelig und war dann noch über mehrere Stunden hinweg unsicher. Danach bildeten sich alle Symptome vollständig zurück.

Prof. Dr. med. H.S. FüeßI

- Ward B, ZeeD (bward15@jhmi.edu).Dizziness and vertigo during MRI. N Engl J Med. 2016;375:e44 